\title{
Mineral Wool Primary Layer Formation in Collecting Chamber
}

\author{
Benjamin Bizjan* - Marko Peternelj - Branko Širok \\ University of Ljubljana, Faculty of Mechanical Engineering, Slovenia
}

The process of the mineral wool primary layer formation was investigated experimentally on a model spinning machine with sucrose as a working medium. The fibers were pneumatically transported from the spinner rotor to the accumulation grid with the aid of the blow-away and the suction airflow. The fiber primary layer formed on the accumulation grid was visualized by a camera for several different operating regimes. Acquired images were post-processed to determine the light absorption in the primary layer, which was then used to calculate the bulk density and the surface density of the layer. Based on the measured process quantities, we were able to form multiple regression models with a relatively good correlation to the experimental data. The models as well as the qualitative image analysis show a significant effect of spinner rotor rotational speed, pneumatic transport velocity and fiber deposit mass on primary layer bulk density and spatial distribution of fibers.

Keywords: mineral wool, fiber, primary layer, spinning machine, collecting chamber, pneumatic transport

Highlights

- Mineral wool primary layer formation was modeled experimentally using molten sucrose.

- $\quad$ Light-absorption visualization approach was used to measure the primary layer density and structure.

- Primary layer structure is significantly affected by the spinner rotational speed, blow-away and suction flow velocity, and the mass of accumulated fibers.

- Multiple regression models for the primary layer density and its standard deviation are in a good agreement with the measurements.

\section{INTRODUCTION}

Mineral wool is a fibrous material commonly used for thermal and acoustic insulation and can be divided into subtypes such as the rock wool and the glass wool. Mineral wool production process is highly complex, consisting of several different production phases. Sufficient understanding and control of each phase is required in order to optimize the properties (e.g. thermal conductivity, homogeneity and mechanical properties) and production costs of the end products.

Production phases which most significantly affect the mineral wool quality are the melt fiberization on the spinning machine, fiber pneumatic transport and primary layer formation in the collecting chamber [1]. Two most commonly used spinner geometries are solid spinning wheels, used mainly for rock wool production, and hollow perforated rotors, used for glass wool production. The melt fiberization process was a subject of several experimental and numerical studies for both solid wheel spinners (Czygany et al. [2], Vad and Morlin [3], Zhao et al. [4]) and perforated rotors (Panda et al. [5], Mukundan and Panda [6], Marheineke and Wegener [7], Marheineke et al. [8], Qin et al. [9] and [10], Kraševec et al. [11]).

Fiber formation phase is followed by pneumatic transport of fibers in the blow-away flow to the collecting chamber where a primary layer of mineral wool is formed [1]. A good quality primary layer is defined by a low degree of spatial fluctuations in thickness and density of the fiber deposit, and is very important for achieving optimal insulation and mechanical properties of the end products. Due to the high complexity and multiphase nature of the flow, experimental investigation of the primary layer formation is difficult and mostly limited to the measurement of aerodynamic characteristics and flow visualization.

The transport of fibers in an axial airflow was modeled numerically by Lin et al. [12] and experimentally by Capone et al. [13] and Qi et al. [14]. In all of these studies, fibers had a relatively low aspect ratio (i.e. length to diameter ratio) and were treated as rigid bodies. On the other hand, mineral wool fibers typically have aspect ratios in excess of 1000 [1], leading to complex 3D flows with mutual intertwining and breakage of fibers. Consequently, the spatial structure of the fiber primary layer deposited on the accumulation grid may show significant inhomogeneities. Also, the newly formed primary layer can be damaged (torn apart and moved) by the flow of the blow-away air onto the accumulation grid. Šrok et al. [1] showed that a reduction in air velocity extremes and gradients on the accumulation grid improves the quality of the mineral wool primary layer as the tearing and redistribution of the layer is largely reduced. The measures proposed so far to improve the velocity distribution include the addition of centrifugal 
rotors to the spinner wheels and modifications to the collecting chamber geometry [1]. However, further optimization and efficient real-time control of the primary layer formation require an accurate model of the process. Due to the process complexity and dependence on many operating parameters, only integral-level models were formed so far [1], proving only partly successful for this purpose.

To overcome the complexity of the industrial mineral wool production environment and the limited range in which the operating parameters can be varied, we have developed a model spinning machine similar to the glass wool spinners. The primary layer was analyzed by a visualization method, and its quality was characterized by multiple regression models.

This paper is organized as follows. The Methods section presents the theoretical background of the mineral wool primary layer formation and the layer visualization method used in our experiments. In the Experimental section, the model spinning machine and the corresponding measurement setup will be introduced whereas the Discussion section will present qualitative and quantitative results of visualization image analysis.

\section{THEORETICAL BACKGROUND}

The primary layer formation process is an important part of the mineral wool production line (see Fig. 1 for an example of a rock wool production line).

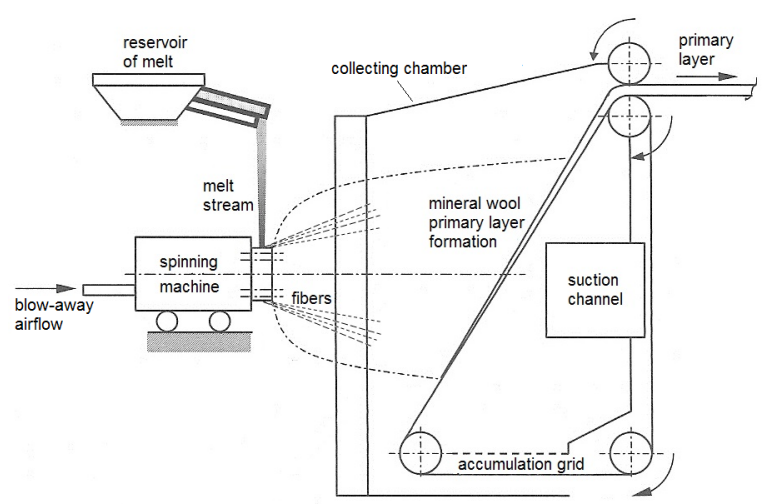

Fig. 1. Rock wool production line between the melting furnace and the primary layer formation zone

Mineral melt is prepared in the melting furnace from which it first enters the melt reservoir and is then supplied to the spinning machine in form of a free falling melt stream. In a rock wool production line, the melt typically impinges on a mantle surface of one or more spinning wheels, forming a film which continuously disintegrates into liquid ligaments. In

manufacturing of glass wool, the fiberization process is different as the melt stream flows inside a spinning perforated rotor, forming a film on its internal mantle surface. Under the action of centrifugal force, melt film is then extruded through the perforations to form ligaments. Nevertheless, the phase following the ligament formation is similar for both spinner designs. Shortly after formation, melt ligaments solidify to fibers and are impregnated by the binder liquid. The fibers then enter the coaxial blow-away airflow (also known as the primary flow) and detach from the spinning machine, forming complex multiphase flow structures. The blow-away flow with fibers enters the collecting chamber while its velocity is reduced from an initial magnitude in excess of $100 \mathrm{~m} / \mathrm{s}$ to below 10 $\mathrm{m} / \mathrm{s}$. Apart from the blow-away flow, the suction flow (also known as the secondary flow) is also present in the collecting chamber to allow the fibers to settle on the accumulation grid and thus form the primary layer. The layer is then compacted by several mechanical processes and is sent to the curing chamber where binder polymerization occurs.

The exact mechanism of the primary layer formation, including the fiber pneumatic transport, breakage and settling on the accumulation grid, is still largely unknown due its complexity and difficulty in observation on smaller length scales. While the primary layer quality control on industrial production lines is still mostly manual [1], there have been several attempts to quantitatively characterize the primary layer quality for the purpose of process automation. The most commonly used measurement method is the camera visualization of the mineral wool primary layer. A sample primary layer image is shown in Fig. 2. A good quality primary layer is characterized by a homogeneous structure of the fiber deposit (Fig. 2a) whereas an inhomogeneous structure with large variations in thickness and density indicates a poor quality of the primary layer.

a)

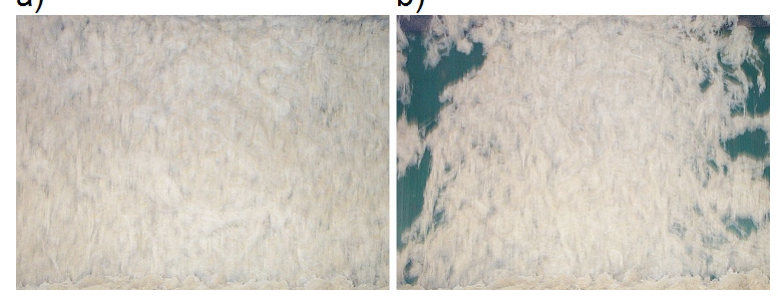

Fig. 2. Primary layer on the accumulation grid; a) homogeneous structure; $b$ ) inhomogeneous structure

Apart from the qualitative analysis, quantitative formulations based on the image gray level can 
be used to analyze the homogeneity and texture of visualization images. Blagojević et al. [15] used a multiple regression model to predict the primary layer homogeneity. The same kind of visualization setup was also used by Bajcar et al. [16] and was followed by a computational fluid dynamics (CFD) analysis of the problem, confirming a significant effect of the local aerodynamic characteristics on the primary layer formation. Apart from the camera visualization, another possible method of primary layer structure analysis is by X-ray scanning, which is used mostly in the glass wool industry [11] and [17], although very little research work has been published so far. The method is based on radiation absorption and allows for the measurement of local primary layer densities, unlike the visualization method used in references [15] and [16], which can only provide information about the layer surface dynamics. To overcome the technical and confidentiality constraints of the industrial environment, a model study using the light absorption method is viable.

Based on the overview of the available literature, the area density $\mu$ and the bulk density $\rho$ were determined to be the most representative primary layer properties in our experiments. To measure $\mu$ and $\rho$, a measurement setup with a visible light camera and lighting source was used so that the fibers were illuminated from behind. Similarly to the X-ray measurement method [11] and [17], which is impractical for small-scale lab experiments, the calculations of $\mu$ and $\rho$ were performed based on the measured light absorption ratio $I / I_{0}$ (Fig. 3).

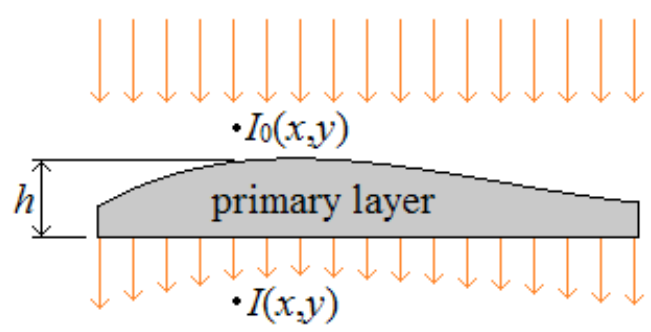

Fig. 3. Light absorption over the primary layer

Local primary layer thickness $h$ (Eq. (2)) at position $(x, y)$ can be obtained from the Beer-Lambert absorption law (Eq. (1)).

$$
\begin{aligned}
& I=I_{0} \exp (-\alpha h), \\
& h=-\frac{1}{\alpha} \ln \left(\frac{I}{I_{0}}\right) .
\end{aligned}
$$

In the next step, the total volume $V$ of the primary layer can be calculated by surface integration of $h$; Eq. (3).

$$
V=\oint_{A} h d A .
$$

For a known primary layer mass $m$ (determined by weighing or other methods), the mean bulk density of the layer $\rho$ can now be calculated as:

$$
\rho=\frac{m}{V} .
$$

Another characteristic primary layer property is the local area density $\mu$ and its normalized standard deviation $\sigma_{\mu n}$ :

$$
\begin{gathered}
\mu=h \rho, \\
\sigma_{\mu n}=\frac{\sigma_{\mu}}{\bar{\mu}}=\frac{\sigma_{\mu}}{m / A} .
\end{gathered}
$$

Alternately, the area density can be calculated directly from the Beer-Lambert law using the mass attenuation coefficient $\alpha_{m}$ (Eq. (7)). However, $\alpha_{m}$ is significantly more difficult to measure than $\alpha$, making such calculation approach impractical unless $\alpha_{m}$ is already known.

$$
\mu=\frac{\ln \left(I_{0} / I\right)}{\alpha_{m}} .
$$

Note that listed calculation procedures assume a stationary accumulation grid. In the case of a moving grid, mass and volume would be replaced with their temporal derivatives (i.e. flows).

\section{EXPERIMENTAL WORK}

Experiments were conducted on a model spinning machine with a hollow perforated rotor and a vertical collecting chamber (Fig. 4a). The spinning machine used was basically a modified version of a cotton candy machine The Breeze 3030EX [18]. The rotor with $70 \mathrm{~mm}$ radius (Fig. 4b) had an integrated heater and was supplied with crystalline sucrose prior to each experiment. During the spinner operation, sucrose was melted by the heater at a rate of approximately 0.75 $\mathrm{g} / \mathrm{s}$ while the rotor rotational speed was regulated by a single-phase variable frequency drive (VFD) in a range between $40 \mathrm{~Hz}$ and $50 \mathrm{~Hz}$. Coaxial blow-away airflow was supplied through 45 circular nozzles (1 $\mathrm{mm}$ diameter, $5 \mathrm{~mm}$ length) evenly distributed around the supply ring on a radius of $80 \mathrm{~mm}$. The blow-away flow was regulated by a valve so that the overpressure in the supply ring $\Delta p$ was between $0 \mathrm{kPa}$ and $50 \mathrm{kPa}$.

The spinning machine was placed inside a vertical collecting chamber with a circular cross section $(0.63$ 
a)

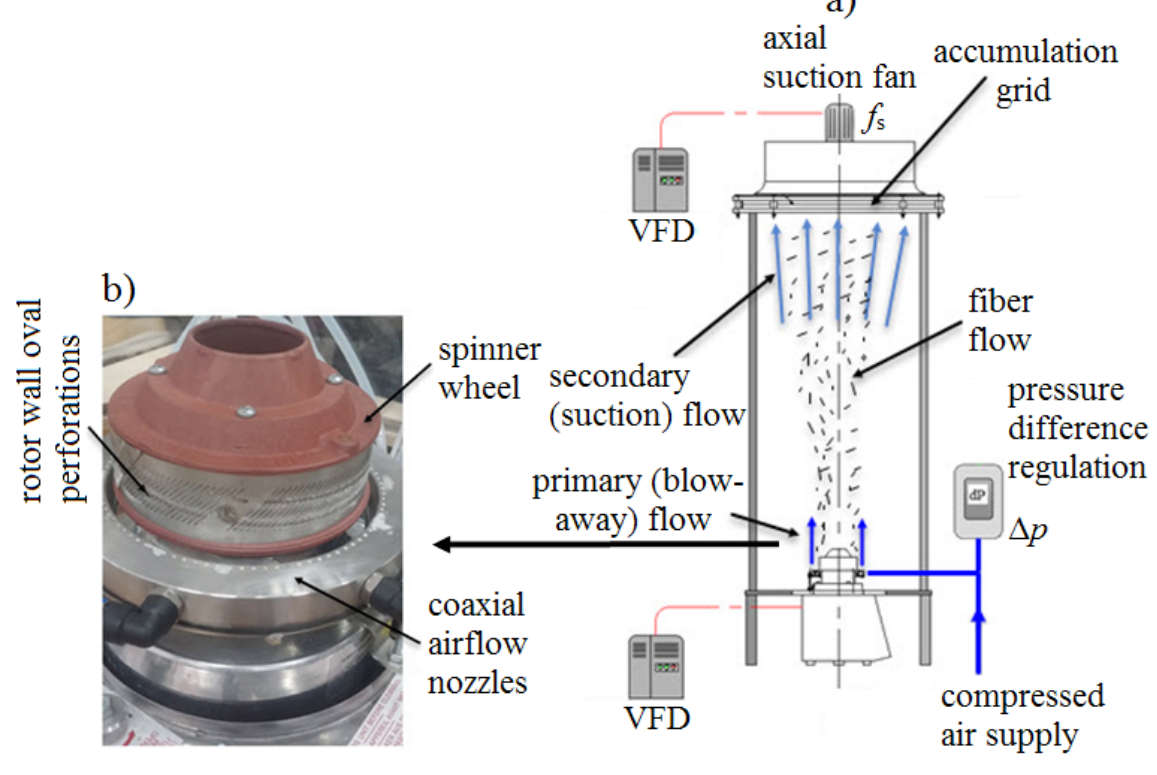

c)
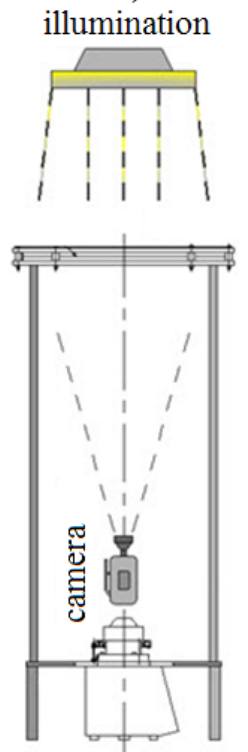

Fig. 4. Experimental setup; a) spinner operation; b) spinner head (detail); c) primary layer visualization

$\mathrm{m}$ in diameter) and a transparent wall. Secondary (i.e. suction) airflow was generated by a 7-blade axial fan powered by a three-phase VFD. The fan was placed on top of the collecting chamber immediately after the horizontal accumulation grid. VFD power supply frequency was varied between $0 \mathrm{~Hz}$ and $40 \mathrm{~Hz}$, which resulted in fan rotational speeds between $0 \mathrm{~Hz}$ and $18.4 \mathrm{~Hz}$.

About 40 seconds after the start of the spinner and air supply operation, fibers started to appear on the spinner rotor and were blown to the collecting chamber. After additional $10 \mathrm{~s}$ to $30 \mathrm{~s}$, the heater was turned off, and upon the end of fiber formation, rotor and airflows were stopped. Then, the fiber primary layer was photographed, removed from the accumulation grid and weighed, with mass being an additional parameter (range between $7 \mathrm{~g}$ and $33 \mathrm{~g}$ ). A list of operating points is provided in Table 1, along with the layer bulk and area density computed by Eqs. (4) and (5), respectively.

Note that the volume flow rates in Table 1 are given for the mean atmospheric conditions during our measurements: $23{ }^{\circ} \mathrm{C}$ temperature, $98.6 \mathrm{kPa}$ absolute pressure and $29 \%$ relative humidity.

In each of the operating points listed in Table 1 , primary layer was visualized as follows. After stopping the spinner operation, the suction fan was removed and the primary layer was illuminated from above by diffuse light. A camera (Casio EX-F1) was
Table 1. Operating points for primary layer visualization

\begin{tabular}{|c|c|c|c|c|c|c|c|c|}
\hline $\begin{array}{c}\text { Op. } \\
\text { point } \\
\text { No. }\end{array}$ & $\begin{array}{c}f_{c} \\
{[\mathrm{~Hz}]}\end{array}$ & $\underset{[\mathrm{kPa}]}{\Delta p}$ & $\begin{array}{c}f_{s} \\
{[\mathrm{~Hz}]}\end{array}$ & $\begin{array}{l}m \\
{[g]}\end{array}$ & $\underset{\left[\mathrm{m}^{3} / \mathrm{h}\right]}{Q_{p}}$ & $\underset{\left[\mathrm{m}^{3} / \mathrm{h}\right]}{Q_{p}}$ & $\begin{array}{c}\rho \\
{\left[\mathrm{kg} / \mathrm{m}^{3}\right]}\end{array}$ & $\begin{array}{c}\sigma_{\mu n} \\
{[\%]}\end{array}$ \\
\hline $1 \mathrm{a}$ & 50 & 50 & 7.0 & 10.5 & 27.0 & 2700 & 2.36 & 192 \\
\hline $1 b$ & 50 & 50 & 7.0 & 14.5 & 27.0 & 2700 & 2.21 & 192 \\
\hline $2 a$ & 50 & 50 & 9.1 & 9.5 & 27.0 & 3600 & 2.05 & 187 \\
\hline $2 b$ & 50 & 50 & 9.1 & 10.5 & 27.0 & 3600 & 2.32 & 200 \\
\hline $2 \mathrm{C}$ & 50 & 50 & 9.1 & 12.5 & 27.0 & 3600 & 2.59 & 213 \\
\hline $3 a$ & 50 & 50 & 11.4 & 9.5 & 27.0 & 4500 & 2.53 & 253 \\
\hline $3 b$ & 50 & 50 & 11.4 & 13.0 & 27.0 & 4500 & 2.57 & 197 \\
\hline $3 c$ & 50 & 50 & 11.4 & 30.0 & 27.0 & 4500 & 3.25 & 123 \\
\hline 4 & 50 & 20 & 7.0 & 10.5 & 17.8 & 2700 & 2.32 & 171 \\
\hline 5 & 50 & 20 & 9.1 & 11.0 & 17.8 & 3600 & 1.97 & 203 \\
\hline 6 & 50 & 20 & 11.4 & 11.0 & 17.8 & 4500 & 1.89 & 189 \\
\hline 7 & 50 & 0 & 7.0 & 11.0 & 0 & 2700 & 2.10 & 200 \\
\hline 8 & 50 & 0 & 9.1 & 11.2 & 0 & 3600 & 2.48 & 126 \\
\hline 9 & 50 & 0 & 11.4 & 13.5 & 0 & 4500 & 1.86 & 147 \\
\hline 10 & 40 & 50 & 7.0 & 12.5 & 27.0 & 2700 & 1.99 & 177 \\
\hline 11 & 40 & 50 & 9.1 & 13.5 & 27.0 & 3600 & 2.00 & 171 \\
\hline 12 & 40 & 50 & 11.4 & 11.5 & 27.0 & 4500 & 2.06 & 196 \\
\hline 13 & 40 & 20 & 7.0 & 9.0 & 17.8 & 2700 & 1.86 & 241 \\
\hline 14 & 40 & 20 & 9.1 & 10.0 & 17.8 & 3600 & 2.38 & 312 \\
\hline $15 a$ & 40 & 20 & 11.4 & 18.0 & 17.8 & 4500 & 2.55 & 182 \\
\hline $15 b$ & 40 & 20 & 11.4 & 12.5 & 17.8 & 4500 & 2.29 & 266 \\
\hline 16 & 40 & 0 & 7.0 & 13.0 & 0 & 2700 & 2.98 & 167 \\
\hline 17 & 40 & 0 & 9.1 & 11.5 & 0 & 3600 & 2.72 & 188 \\
\hline 18 & 40 & 0 & 11.4 & 6.0 & 0 & 4500 & 2.18 & 212 \\
\hline 19 & 40 & 50 & 0 & 11.5 & 27.0 & 0 & 2.63 & 166 \\
\hline 20 & 40 & 50 & 18.4 & 33 & 27.0 & 7200 & 6.91 & 117 \\
\hline
\end{tabular}


placed underneath the accumulation grid and an image of the layer was taken (Fig. 5) using manual aperture and shutter time settings $(f / 7.5$ and $0.08 \mathrm{~s}$, respectively). For reference, the background (grid without fibers) was also photographed using the same visualization setup. Images were then processed by the method presented in section 1 so that the bulk and area density (Fig. 5b) was calculated. Also, the maximum thickness of the primary layer was manually measured (Fig. 5c) and used in Eq. (1) to obtain the attenuation coefficient $\alpha$. The value $\alpha \approx 0.17 \mathrm{~m}^{-1}$ was obtained for all operating points (OP) except OP 20, where $\alpha=0.35$ $\mathrm{m}^{-1}$ was determined. This was most likely due to the more compact primary layer in OP 20 caused by a much higher suction flow rate.
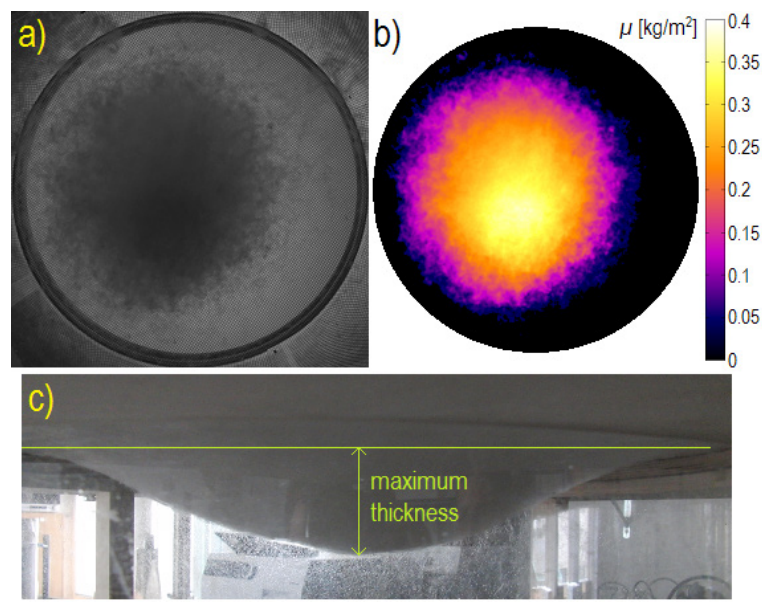

Fig. 5. Sample visualization images (OP 3c); a) raw image; $b$ ) area density field; c) primary layer side view

\section{RESULTS AND DISCUSSION}

\subsection{Qualitative and Quantitative Properties of the Primary Layer}

Spinning machine operating parameters were varied in a wide range (Table 1) and produced distinct forms of the sucrose primary layer (Fig. 6).

Primary layer mass distribution in Fig. 6 shows a significant effect of all operating parameters varied in the experiments. An increase of the spinner rotational speed (OP 13 and 4 represent $f_{c}=40 \mathrm{~Hz}$ and $f_{c}=50$ $\mathrm{Hz}$, respectively) increases the primary layer area due to the larger centrifugal forces which widen the fibrous flow. At the same time, the layer becomes more uniform (note a reduction in $\sigma_{\mu n}$; Table 1).

An even greater effect on the primary layer structure can be seen with regard to the airflow characteristics. In Fig. 6, the effect of the blow-

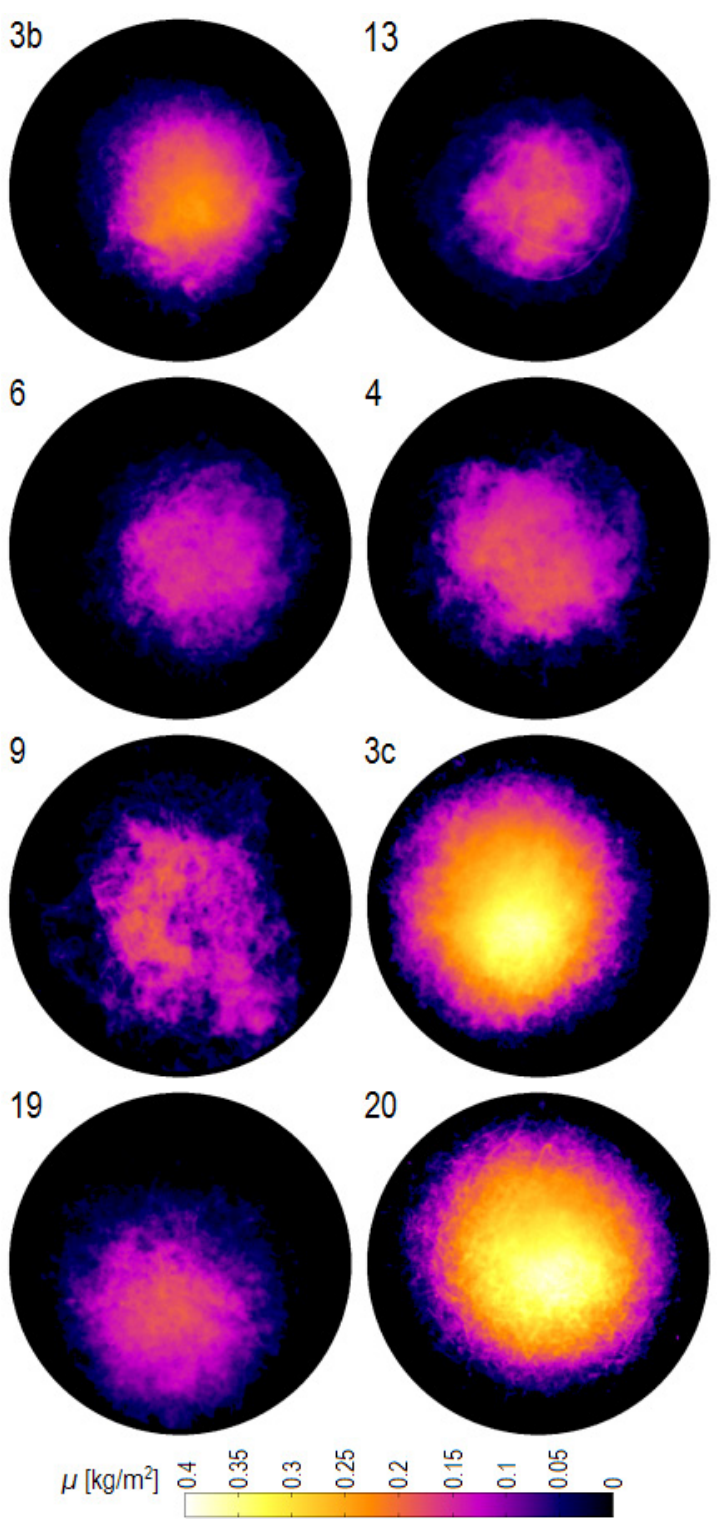

Fig. 6. Fields of primary layer area density for different operating points; given are the OP numbers

away pressure (and consequently, flow rate) is seen by comparison of plots for OP 9, 6 and $3 b$, where $\Delta p$ was $0 \mathrm{kPa}, 20 \mathrm{kPa}$ and $50 \mathrm{kPa}$, respectively. When the fibers are transported only by the suction flow (OP $9, \Delta p=0, Q_{p}=0$ ), the primary layer is asymmetrically shaped and scattered across the large portion of the accumulation grid. In this operating mode, large chunks of fiber wool were observed on the grid, leading to an unwanted inhomogeneity in the layer structure. As the blow-away pressure was raised towards $50 \mathrm{kPa}$ (OP 4 and $3 \mathrm{~b}$ ), the primary layer shape became more axissymmetric and with fewer 
visible inhomogneities. However, the layer became pile-shaped with a large thickness in the center, and its mean bulk density slightly increased. Such pile formation is unfavorable as the layer thickness should be as uniform as possible, but could largely be avoided by using a moving mesh.

Apart from the primary flow, the secondary (suction) flow also has a large effect on the quality of the primary layer. When the suction flow is not present and pneumatic transport depends entirely on the blow-away flow (OP 19, $f_{s}=18.4 \mathrm{~Hz}$ ), the fibers may still reach the accumulation grid, but the flow is unstable and the primary layer can form far from the grid center. An increase in the suction flow rate, as clearly shown by data for the operating points 4 and $6\left(f_{s}=7.0 \mathrm{~Hz}\right.$ and $f_{s}=11.4 \mathrm{~Hz}$, respectively), causes the primary layer to shrink in area while its bulk density increases. If the suction flow rate is further increased (OP 20, $\left.f_{s}=18.4 \mathrm{~Hz}\right)$, the layer bulk density rapidly increases while the area density distribution becomes practically axissymmetric. This is partly due to the fact that at larger $Q_{s}$, the pressure drop over the primary layer rises, resulting in a more intense layer compression.

Another contributing factor to the large bulk density in OP 20 was a relatively large primary layer mass $(m=33.0 \mathrm{~g})$, which, due to the larger quantity of fibers, reduced the permeability of the accumulation grid and thus further increased the pressure drop over the layer. In OP $3 \mathrm{c}$ where the mass of the primary layer is similar $(m=30.0 \mathrm{~g})$, the spatial distribution and maximum of $\mu$ is comparable to OP 20 , but the bulk density is about $50 \%$ lower, meaning that the layer is much less compact. For comparison, the layer bulk density in operating points $3 \mathrm{a}, 3 \mathrm{~b}$ and $3 \mathrm{c}$ only varies by $28 \%$ despite the mass range of approximately $1: 3$ ( $\rho$ rises monotonously with $m$, though). This suggests that the bulk density more sensitive to the suction flow rate than the primary layer mass, but both parameters are significant.

Due to the specific experimental setup (i.e. stationary accumulation grid) it is important to note that the primary layer formation is a transient process. The layer mass (or equally, fiber deposition time) is analogous to the combination of the fiberization mass flow and the accumulation grid velocity in a typical mineral wool manufacturing process.

\subsection{Multiple Regression Models}

Based on the results presented in the previous subsection, we suspect a strong correlation between the characteristic properties of the primary layer $(\rho$ and $\sigma_{\mu n}$, Table 1) and the process input parameters $\left(f_{c}\right.$, $\left.Q_{p}, Q_{s}, m\right)$. This hypothesis will be confirmed by the following power law multiple regression models:

$$
\begin{gathered}
\rho=a_{0} \cdot f_{c}^{a_{1}} \cdot Q_{p}^{a_{2}} \cdot Q_{s}^{a_{3}} \cdot m^{a_{4}}, \\
\sigma_{\mu n}=a_{0} \cdot f_{c}^{b_{1}} \cdot Q_{p}^{b_{2}} \cdot Q_{s}^{b_{3}} \cdot m^{b_{4}} .
\end{gathered}
$$

The exponents $a_{0}$ to $a_{4}$ and $b_{0}$ to $b_{4}$ were determined by fitting of the power law models in Eqs. (8) and (9) to data in Table 1 (data was inserted in units given in the Nomenclatures section). Operating points with $Q_{p}=0$ or $Q_{s}=0$ were excluded from the model to avoid singularities. These points were only intended to demonstrate the most extreme operating conditions unsuitable for an industrial manufacturing process.

For Eq. (8) we obtain $a_{0}=112, a_{1}=-0.141$, $a_{2}=0.225, a_{3}=0.389, a_{4}=0.498$ and a relatively high coefficient of determination $\left(R^{2}=0.88\right)$. This indicates a good correlation between modeled and input parameters.

For Eq. (9), the following values were calculated: $b_{0}=14.2, \quad b_{1}=-0.842, \quad b_{2}=0.262, \quad b_{3}=0.116$, $b_{4}=-0.589$ and a $R^{2}=0.78$. The $R^{2}$ value is sufficiently high to conclude that the $\sigma_{\mu n}$ statistical parameter is representative of the process quality and can be modeled from selected input parameters.

Regression models can now be rewritten in a final form:

$$
\begin{gathered}
\rho=112 \cdot f_{c}^{-0.141} \cdot Q_{p}^{0.225} \cdot Q_{s}^{0.389} \cdot m^{0.498}, \\
\sigma_{\mu n}=14.2 \cdot f_{c}^{-0.842} \cdot Q_{p}^{0.262} \cdot Q_{s}^{0.116} \cdot m^{-0.589} .
\end{gathered}
$$

The goodness of fit of these regression models can also be shown graphically, Figs. 7 and 8. It is evident that most of the measurements are in a good agreement with the model.

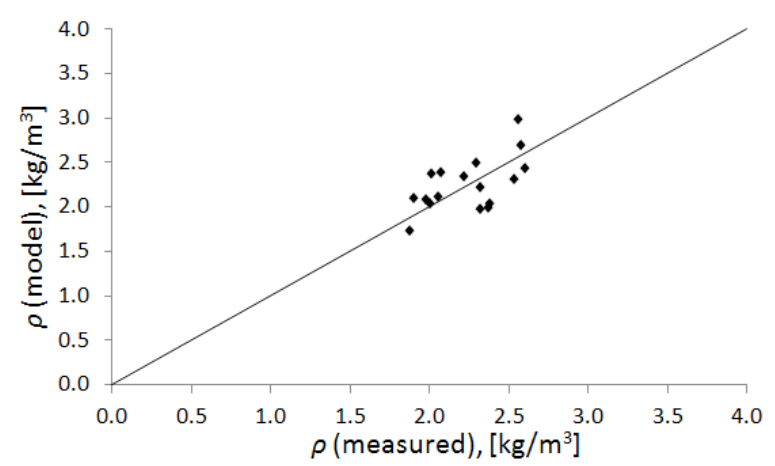

Fig. 7. Correlation between measured and modeled values of $\rho$

The model in Eq. (10) indicates that the bulk density of the primary layer increases with the flow 
rate of the blow-away and suction air as well as with the layer mass. As said, this can be explained by layer compression due to increased pressure drop over the accumulation grid. On the other hand, $\rho$ falls with rotational speed of the spinner rotor as fiber centrifugal forces are increased, scattering fibers to a larger area. Consequently, the layer thickness is reduced, leading to a reduction of the pressure drop across the accumulation grid.

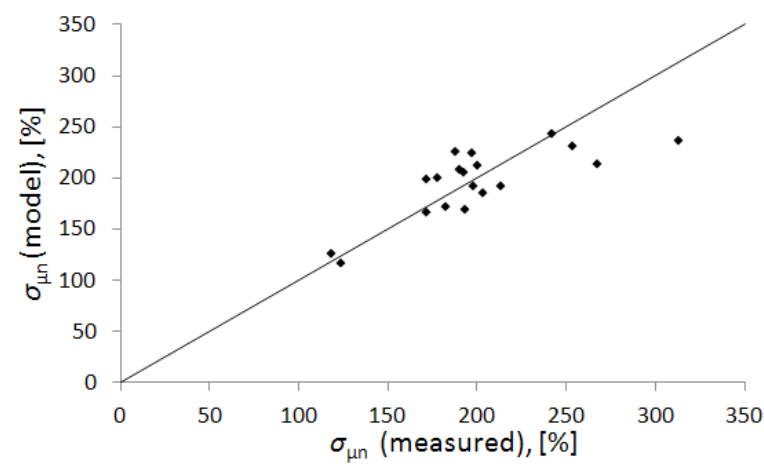

Fig. 8. Correlation between measured and modeled values of $\sigma_{\mu n}$

In comparison with Eq. (10), the exponent values in the model for $\sigma_{\mu n}$ (Eq. (11)) are very different. $\sigma_{\mu \mathrm{n}}$ decreases with the spinner rotational speed, which can again be explained by the scattering of the fibers over a larger area, resulting in a primary layer of a more uniform thickness. However, $\sigma_{\mu n}$ also decreases with the primary layer mass as the layer structure becomes more and more homogenous when it grows in thickness. On the other hand, $\sigma_{\mu n}$ seems to slightly rise with both blow-away and suction flow rate. This is most likely due to the fact that an increase in $Q_{p}$ and $Q_{s}$ results in a more axissymmetric primary layer formation. This may (under certain conditions) contribute to formation of piles on the accumulating grid, and $\sigma_{\mu n}$ increases due to variations in the primary layer height rather than structural inhomogeneities.

\section{CONCLUSIONS}

The process of the mineral wool primary layer formation was studied experimentally on a model spinning machine with sucrose as a working medium. The bulk and area density of the layer were determined by a light absorption method which provided a good measure for the layer structure and thickness. The bulk density, which should ideally be as low as possible, was found to depend on the spinner rotational speed, flow rates of the blow-away and suction air as well as well as the mass of accumulated fibers. The bulk density of the primary layer was shown to increase with its own permeability until a new equilibrium is reached. Another important measure for the primary layer is the homogeneity of its structure which was quantified by the normalized standard deviation of the layer area density, $\sigma_{\mu n}$. A low value of $\sigma_{\mu n}$ is desired as it implies a good uniformity of the primary layer thickness and structure.

An important step in optimization of investigated process is to determine an envelope of the operating parameters which yields a good quality primary layer. The spinner rotational speed was shown to control the layer width and height profile while the size and spatial distribution of inhomogeneities is mostly determined by the pressure and flow rate of the blowaway airflow. On the other hand, the primary layer bulk density is most significantly affected by the suction flow rate and layer mass. Further research should include the modeling of an actual mineral wool manufacturing process with a moving accumulating grid where primary layer formation is continuous. New experimental and numerical models will improve the understanding of the fiber transport and primary layer formation process and introduce new possibilities for automatization of the process control.

\section{NOMENCLATURES}

\begin{tabular}{|c|c|c|}
\hline$A$ & {$\left[\mathrm{~m}^{2}\right]$} & accumulation grid surface \\
\hline$f_{c}$ & {$[\mathrm{~Hz}]$} & spinner rotor rotational speed \\
\hline$f_{s}$ & {$[\mathrm{~Hz}]$} & suction fan rotational speed \\
\hline$h$ & {$[\mathrm{~m}]$} & primary layer height \\
\hline$I$ & {$\left[\mathrm{Wm}^{-2}\right]$} & light intensity after absorption \\
\hline$I_{0}$ & {$\left[\mathrm{Wm}^{-2}\right]$} & incoming light intensity \\
\hline$m$ & {$[\mathrm{~kg}]$} & primary layer mass \\
\hline$Q_{p}$ & {$\left[\mathrm{~m}^{3} \mathrm{~s}^{-1}\right]$} & blow-away flow rate \\
\hline$Q_{s}$ & {$\left[\mathrm{~m}^{3} \mathrm{~s}^{-1}\right]$} & suction flow rate \\
\hline$V$ & {$\left[\mathrm{~m}^{3}\right]$} & primary layer volume \\
\hline$x$ & {$[\mathrm{~m}]$} & distance from left image edge \\
\hline$y$ & {$[\mathrm{~m}]$} & distance from top image edge \\
\hline$\alpha$ & {$\left[\mathrm{m}^{-1}\right]$} & attenuation coefficient \\
\hline$\alpha_{m}$ & {$\left[\mathrm{~m}^{2} \mathrm{~kg}^{-1}\right]$} & s attenuation coefficient \\
\hline$\Delta p$ & {$[\mathrm{~Pa}]$} & blow-away air overpressure \\
\hline$\mu$ & {$\left[\mathrm{kgm}^{-2}\right]$} & prim. layer mean area density \\
\hline $\bar{\mu}$ & {$\left[\mathrm{kgm}^{-2}\right]$} & prim. layer local area density \\
\hline$\rho$ & {$\left[\mathrm{kgm}^{-3}\right]$} & primary layer bulk density \\
\hline$\sigma_{\mu}$ & {$\left[\mathrm{kgm}^{-2}\right]$} & standard deviation of $\mu$ \\
\hline$\sigma_{\mu \prime}$ & 5 & normalized st. deviation of $\mu$ \\
\hline
\end{tabular}

\section{REFERENCES}

[1] Širok, B., Blagojević, B., Bullen, P. (2008). Mineral Wool: Production and Properties. Woodhead Publishing, Cambridge, DOI:10.1533/9781845694456. 
[2] Czygany, T., Vad, J., Kornel, P. (2005). Basalt fiber as a reinforcement of polymer composites. Periodica Polytechnica Mechanical Engineering, vol. 49, no. 1, p. 3-14.

[3] Vad, J., Morlin, B. (2007). Fluid mechanical model for formation of mineral wool fibers applied in polymer composites. Materials Science Forum, vol. 537-538, p. 269276, DOl:10.4028/www.scientific.net/MSF.537-538.269.

[4] Zhao, D., Zhang, Z., Liu, L., Wang, X. (2015). A novel kinematic model for molten slag fiberization: Prediction of slag fiber properties. Metallurgical and Materials Transactions $B$, vol. 46, no. 2, p. 993-1001, D0l:10.1007/s11663-014-0259-9.

[5] Panda, S., Marheineke, N., Wegener, R. (2008). Systematic derivation of an asymptotic model for the dynamics of curved viscous fibers. Mathematical Methods in Applied Sciences, vol. 31, no. 10, p. 1153-1173, D0I:10.1002/mma.962.

[6] Mukundan, D.V., Panda, S. (2013). Temperature effects on curved stationary viscous thermal fiber jets. Australian Journal of Basic and Applied Sciences, vol. 7, no. 7, p. 613-622.

[7] Marheineke, N., Wegener, R. (2009). Asymptotic model for the dynamics of curved viscous fibres with surface tension. Journal of Fluid Mechanics, vol. 622, p. 345-369, DOI:10.1017/S0022112008005259.

[8] Marheineke, N., Liljo, J., Mohring, J., Schnebele, J., Wegener, R. (2012). Multiphysics and multimethods problem of rotational glass fiber melt-spinning. International Journal of Numerical Analysis and Modeling, Series B, vol. 3, no. 3, p. 330-344.

[9] Qin, Y., Lv, X., Bai, C., Chen, P., Qiu, G. (2013). Dry granulation of molten slag using a rotating multi-nozzle cup atomizer and characterization of slag particles. Steel Research International, vol. 84, no. 9, p. 852-862, D0l:10.1002/ srin.201200325.

[10] Qin, Y., Lv, X., Bai, C., Chen, P., Qiu, G., Jie, Z. (2014). Mechanism of dry molten slag granulation using a rotating multi-nozzle cup atomizer. Steel Research International, vol. 85, no. 1, p. 44-52, D0l:10.1002/srin.201300007.
[11] Kraševec, B., Širok, B., Bizjan, B., Hočevar, M. (2015). Fibre density distribution in a layer of glass wool. Glass Technology - European Journal of Glass Science and Technology, Part A, vol. 56, no. 5, p. 145-152, D0l:10.13036/17533546.56.5.145.

[12] Lin, J.Z., Liang, X.I., Zhang, S.L. (2012). Numerical simulation of fiber orientation distribution in round turbulent jet of fiber suspension. Chemical Engineering Research and Design, vol. 90, no. 6, p. 766-775, D0l:10.1016/j.cherd.2011.09.016.

[13] Capone, A., Romano, G.P., Soldati, A. (2015). Experimental investigation on interactions among fluid and rod-like particles in a turbulent pipe jet by means of particle image velocimetry. Experiments in Fluids, vol. 56, no. 1, Dol:10.1007/s00348014-1876-4.

[14] Qi, G., Nathan, J.G., Lau, C.W.T. (2015). Velocity and orientation distributions of fibrous particles in the near-field of a turbulent jet. Powder Technology, vol. 276, p. 10-17, DOI:10.1016/j.powtec.2015.02.003.

[15] Blagojević, B., Širok, B., Hočevar, M. (2003). Monitoring and control of quality of the primary layer of mineral wool on a disc spinning machine. Instrumentation Science \& Technology, vol. 31, no. 1, p. 67-80, Dol:10.1081/Cl-120018408.

[16] Bajcar, T., Blagojević, B., Širok, B., Dular, M. (2007). Influence of flow properties on a structure of a mineral wool primary layer. Experimental Thermal and Fluid Science, vol. 32, no. 2, p. 440-449, D0I:10.1016/j.expthermflusci.2007.05.007.

[17] Badel, E., Letang, J.-M., Peix, G., Babot, D. (2003). Quantitative microtomography: measurement of density distribution in glass wool and local evolution during a one-dimensional compressive load. Measurement Science and Technology, vol. 14, no. 4, 410-420, Dol:10.1088/0957-0233/14/4/302.

[18] The Breeze Instruction Manual: Models 3030, 3030SR, 3030EX, 3040, 3040SR (2015). From https://www. gmpopcorn.com/products/manuals/42147.pdf, accessed on 2015-08-25. 